Mini Review

\title{
Application of Carbon Based Material for the Electrochemical Detection of Heavy Metal Ions in Water Environment
}

\author{
Guobin Chen ${ }^{1}$, Xiaojun Wang ${ }^{2, *}$, Lili Wang ${ }^{1}$ \\ ${ }^{1}$ Chongqing Key Laboratory of Spatial Data Mining and Big Data Integration for Ecology and \\ Environment, Rongzhi College of Chongqing Technology and Business University, Chongqing \\ 401320, PR China \\ ${ }^{2}$ College of Life Sciences, Nanjing Normal University, Nanjing, 210023, RP China \\ *E-mail: wangxiaojun0910@njnu.edu.cn
}

doi: $10.20964 / 2020.05 .64$

Received: 5 Janaury 2020 / Accepted: 19 February 2020 / Published: 10 April 2020

\begin{abstract}
In recent years, the preparation and application of carbon materials and their composites have been widely studied, and remarkable progress has been made in the detection of environmental pollutants, especially in the study of heavy metal analysis methods in water. In this paper, the research status of various kinds of carbon materials used in the detection of heavy metal ions in water is reviewed, and the preparation, performance, mechanism, advantages and disadvantages of the electrochemical detection methods with carbon material-modified electrodes are categorically analysed. The excellent electron transport properties of carbon materials give them a natural advantage in the development of electrochemical test methods for heavy metal ions that are conducive to the online, in situ and real-time detection of heavy metal ions in water. However, research on carbon-modified electrodes is just in its infancy, and there are some problems, such as poor anti-interference ability and selectivity, poor electrode reusability, and less practical research applications. It is necessary to continue the development and preparation of new composite electrodes that are modified with carbon materials to further improve the selectivity and anti-interference ability of electrochemical testing methods, increase the service life of electrodes and research applications in unconventional environments, and expand the application scope of graphene-based composite electrodes.
\end{abstract}

Keywords: Ecological environment; Carbon based material; Electrochemistry; Heavy metal ions; Composite

\section{$\underline{\text { FULL TEXT }}$}

(C) 2020 The Authors. Published by ESG (www.electrochemsci.org). This article is an open access article distributed under the terms and conditions of the Creative Commons Attribution license (http://creativecommons.org/licenses/by/4.0/). 